\title{
Tentative Analysis on the Characteristics of Physical Training of Tennis Sport
}

\author{
Yonghua Liao \\ University of Electronic Science and Technology of China, Chengdu Sichuan, 611731, China
}

Keywords: Tennis sport, Physical training, Characteristics

\begin{abstract}
As an antagonistic sport with high exercise intensity within a short time, the tennis sport is characterized by the combination of intermittent exercise and rest, and focuses on the player's physical ability, which is used as the key influence factor to enhance the player's whole skill level. Therefore, both the coach and player pay high attention to the physical training of tennis sport. This paper analyzes the combination of tennis sport, connotation of physical training and the project characteristics of tennis sport and explores corresponding training measures by with reference to the characteristics of physical training of tennis sport in the hope of promoting the player's physical quality accordingly and propelling the better development of tennis sport in China.
\end{abstract}

\section{Introduction}

In the tennis competition race, a player needs to not only have good fighting ability with skills and tactics but also compete with the opponent in the physical condition, intelligence and psychological quality. Only when players have excellent physical condition, can they guarantee the full play of their technical and tactical level in the fierce competitive race and thus correspondingly guarantee the victory in the competition. Thus, for tennis players, exploring the characteristics of physical training and conducting scientific physical training by combining the characteristics are of realistic significance for strengthening players' ability and acquiring sports advantages in a competitive race.

\section{Tennis sport and physical training}

\section{Tennis sport}

Specifically, the tennis sport is a continuously fierce and intermittent sports event, which is composed of multiple continuous short-time explosive movements and shows the distinctly high-strength and high-density movement characteristics. As for the essential attribute, the tennis sport can be concluded into the skill-oriented category of net competitive event. It is required that players must keep a good physical condition when participating in competitive activities, have excellent physical ability and sports duration ability, and can obtain certain competitive advantages in the race.

\section{Physical training}

In sports activities, the physical training usually includes two important forms, which are respectively the generalized training and specialized training. The generalized physical training specifically refers to that players cultivate the basic athletic ability of human body through corresponding training and is a basic part of players' competitive skills [1]. The specialized physical training mainly refers to that players' bodies show the powerful athletic ability after professional training for a long time, and determines whether players can obtain corresponding competitive advantages in the special competitive race to some degree so as to win the match. Physical training for players is designed to properly adjust players' physical structural form and all functions by adopting scientific training methods so that the physical training will be more suitable for players' development need and will exert certain positive influences on comprehensively strengthening players' athletic ability. 


\section{Project characteristics of tennis sport}

The tennis sport is characterized by the relatively meticulous, complicated and changeable techniques and tactics. There is an intense competition between opponents in a tennis game. According to the thought of event-group theory proposed by experts concerned, in essence, tennis is a skill-oriented antagonistic net-separating sport, so techniques and tactics have decisive influences on the result of the tennis sport. However, with the booming of competitive sports in current society, both techniques and tactics of tennis sport are continuously innovated and strengthened, so physical ability has an increasingly strong influence on the victory or defeat in sport. Therefore, on the background of current sports development, physical ability is the foundation to guarantee players' all skills in the competitive sport and tactics have the most direct and crucial influences on the result of a sport match, so the physical training of tennis sport has become one of the most key links for tennis development,

\section{Energy supplying characteristics of tennis sport}

As for the analysis regarding the energy supplying system, the tennis sport is generally composed of multiple small antagonistic rounds, each of which is approximately limited to 10 seconds, so the exercise intensity is rather high and fierce and the highest exercise frequency can even reach 200 times per minute. For this reason, ATP-CP is mostly used as the energy supplying system of tennis sport. When the antagonistic round lasts for more than 10 seconds, as driven by the body, the glycolysis energy supplying system in the body of player will participate in the whole energy supplying system to promote the accumulation of lactic acid in a body; when a player takes a rest between rounds, the energy supplying system is dominated by aerobic energy supply to properly eliminate the lactic acid accumulated in the body and to guarantee that the player can recover strength timely. Thus, for good development, tennis players must guarantee to have the most basic explosive force, aerobic exercise ability and glycolysis ability etc. to provide corresponding guarantees for the strengthening and enhancement of comprehensive athletic ability. Meanwhile, as the main energy supplying system within the body of a player, ATP-CP can well supply energy to players during exercise within 6 to 8 seconds, help players to obtain explosive quick movement energy in exercise, even reduce players' absorption of oxygen, and correspondingly affect the reinforcement of players' sports skills [2]. The characteristic of glycolysis energy supplying system is that glycolysis reacts to generate lactic acid within the period of 30 seconds to 2 minutes and sufficient oxygen from the player's respiratory tract is not needed. The aerobic exercise specifically means to guarantee the player to take an oxygen exercise for a long time and corresponding kinetic energy can be provided by the player's own carbohydrate and fat oxidation. Energy supply needs to be completed in the environment with sufficient oxygen.

\section{Kinematics analysis on tennis techniques}

Through the kinematics dissection and analysis on technical movements of contemporary tennis of China, we can specifically determine the muscles with greater functions in tennis sport and thus can make targeted specialized training plans by combining the physical development need of players. Generally, players' muscle groups for professional training in tennis sport are as follows: a series of muscle groups in the forehand side such as kicking with heel, turning waist, medial rotation and forearm pronation; in the backhand side, the muscle groups such as kicking with heel, turning waist, lateral rotation and wrist flexion; the muscle groups for serving are mainly kicking with heel, waist and back, hipbone bending and abdominal curl, elbow stretching, forearm pronation and wrist flexion. To carry out specialized tennis training based on the practical condition of muscle group has a corresponding positive influence on strengthening of tennis players' physical ability.

\section{Characteristics and countermeasures of physical training of tennis sport}

Physical training for tennis sport includes the contents in many aspects such as specialized speed training, sensibility training, endurance training and strength training. Besides, each kind of training 
mode includes its personalized features and needs to be supported by scientific training measures, so scientific analysis on the features and measures for tennis players' participation in physical training can exert corresponding positive influences on the cultivation of players' comprehensive quality and the reinforcement of kinetic competitive ability. Next, this paper will analyze the representative formal features and countermeasures of physical training in tennis sport so as to lay a solid foundation for the strengthening of overall competitive ability of tennis sport.

\section{Features of specialized speed training for tennis sport and implementation measures}

Under the influence of booming of competitive sports in modern society, the tennis confrontation is fiercer and there is even a tendency of offensive tennis. In such a competitive race with an extremely high intensity, players must highly focus on the race, make rapid judgments and physical reactions for the opponent's behaviors, and thus catch the tennis and fight back. In the tennis competitive race, the specialized speed specifically refers to that the players rapidly fight back and react based on the opponent' behavior in the practical sport so as to maximally win scores [3]. As a general rule, a player's one-shot speed is mainly influenced by the following factors, which are respectively anticipation, formal judgment, reaction, response action etc. In this process, a player's reaction speed is affected by the excitement degree, flexibility and conditional reflex consciousness ability of central nervous system. The first two influence factors can be improved through proper specialized training to guarantee the player's physical quality and ability etc. A player's movement speed mainly includes moving and stroke, both of which are affected by the player's muscle strength and instant explosive force. Therefore, to correspondingly strengthen and improve the overall level of tennis competitive sport, it is required to intensify the speed training and train players' reaction speed and moving speed, gradually enhance the human body's nervous system's response ability in all competitive scenes, and optimize the coordination skills of players' muscle groups so as to intensify the overall tennis competitive ability. For instance, in the process of training tennis players' reaction speed, taking a pass at random directions can be used as the training point. Let a student stand on the opposite net-separating line to serve balls to all directions; when the ball bounces, the other student on the other side of the net should make a rapid reaction and maximally hit the ball back to intensify the training of reaction ability. In addition, in this training pattern, students should exchange their roles and adjust the serve rhythm after they are proficient so that the training effect can be maximally exerted.

\section{Features and countermeasures of specialized strength training for tennis sport}

The strength training, which is an important part of the physical training of tennis players, also exerts extremely important influences on the players' overall competitive skills. According to the varied modes of representation of tennis players' strength quality, the strength can be specifically divided into the maximal strength, strength endurance and fast strength etc. The explosive force training in fast strength is the key point in the tennis players' physical quality and has a decisive influence on a tennis match [4]. As shown in relevant investigations, in the studies on the physical quality of Chinese female tennis players, strength plays a significant role in speed, endurance, flexibility and other comprehensive qualities. Meanwhile, since strength quality is the basis for a tennis player to acquire other comprehensive qualities, to train and cultivate the players' strength quality also promotes the enhancement of other comprehensive qualities and correspondingly guarantees players' physical health in a competitive race. Therefore, with the increasing influence of players' physical ability on the result of a competition, it is extremely crucial to carry out corresponding strength training for tennis players.

As for strength training of tennis sport, because players need corresponding abdominal muscle contraction to achieve the upward and forward strength transfer in each stroke in the practical sports process, players can choose a proper sit-up way to train the strength and endurance of their abdominal muscle by combining the features of the sport so as to guarantee to maximally give play to the function of abdominal muscle group during practical strokes and to improve the competition level. As for the hand and shoulder strengths which also exert important influences on strokes, to complete powerful and continuous strokes in a match and to burst out powerful strength in a short time, players 
can try to use press-ups to train the upper muscle of their bodies to improve the muscle toughness and contractility, to intensify players' overall competitive ability and, meanwhile, to protect the muscle against strains.

\section{Features and countermeasures of physical sensitivity and flexibility training of tennis player}

Before hitting a ball, tennis players need to properly adjust their bodies, keep the best stroke status and have good physical stabilizing ability and flexibility in the process so that they can correctly judge the drop points of ball, speed and return stroke lines etc. so as to finally win the match. For this reason, in the process of implementing physical training for players, it also required to properly attach important to the sensitivity quality and flexibility quality, correspondingly guarantee the sensitivity and flexibility based on strength and speed trainings, and intensify and improve the overall competitive ability of tennis players. It is found through relevant studies that human body's coordination skills are affected by the congenital factors but can also be largely improved through arduous training; besides, the aspects and degree of enhancement are affected by the types of training projects and training intensity. As for players, the good coordination skills generated based on high physical sensitivity and flexibility can help players to rapidly command the technical key points when learning the professional techniques and tactics, to reach the best stroke effect based on their physical quality and to maximally reduce the loss caused to the whole match due to players' faults of action. The improvement in players' physical flexibility can properly strengthen the flexibility of joints, further enhance the strength and speed of driving balls and serving balls and correspondingly guarantee their health in competitive activities. However, it is needed to be noticed in this quality training process that excessively intensified physical flexibility of tennis players will have a negative impact on the development of their physical quality [5]. Thus, to ensure the best condition for the physical quality of players, it is also required to control the coordination of all physical trainings to guarantee maximally improving the competitive level of players who participate in scientific training. With respect to the practical training for the two qualities, for intensified training of sensitivity quality, a proper way of changing movements can be chosen according to the practical condition. Players are required to immediately do or stop an action according to the command to improve their reaction ability. Regarding the cultivation of flexibility, players can participate in proper trainings for different body parts. For instance, organize players to choose "pressing shoulder with body forward", "body forward with straight legs", "lying flat on the stomach with the back upward" and many other training modes for targeted training to intensify their physical quality.

\section{Conclusion}

To sum up, physical training plays a crucial role in enhancing the overall tennis training effect and is the foundation for gradual intensifying tennis players' physical quality and competitive level. Therefore, personnel concerned should strengthen the studies on tennis players' training for promotion, timely optimize and adjust the training mode in accordance with the training features and practical needs, promote the maximal effects of training, and also propel the development of tennis industry of China based on intensifying players' competitive ability.

\section{References}

[1] Zhuang Jing. Brief Discussion on the Physical Training of Tennis Sport. Bulletin of Sport Science \& Technology, 2013, 21 (1):38-41

[2] Zhang Yongduo. Study on Concept and Methods of Physical Training of Tennis Sport for College Students. Contemporary Sports Technology, 2014, 4 (23):28, 30.

[3] Li Baohai. Study on Methods for Physical Training of Tennis Sport for College Students. Sport Time, 2015 (4):127-127.

[4] Gu Da. Study on Features of Physical Training of Competitive Tennis Event. Contemporary Sports Technology, 2014, 4 (1): 28, 30. 
[5] Sun Lidong. Methods for Physical Training of Modern Tennis. Sport Time, 2013 (9):133. 